\title{
Effects of genotype on the biomechanical parameters and composition of bone in the laying hen embryos
}

\author{
Fatma Kübra ERBAY ELIBOL ${ }^{1, a, \unrhd}$, Esin Ebru ONBAŞILAR ${ }^{2, b}$, Tuğba KARAKAN ${ }^{3, c}$, \\ Süleyman TABAN ${ }^{4, \mathrm{~d}}$, Teyfik DEMİR ${ }^{5, \mathrm{e}}$
}

\begin{abstract}
${ }^{1}$ TOBB University of Economics and Technology, Department of Biomedical Engineering, Ankara; ${ }^{2}$ Ankara University, Faculty of Veterinary Medicine, Department of Animal Breeding and Husbandry, Ankara; ${ }^{3}$ Ankara University, Faculty of Veterinary Medicine, Department of Animal Nutrition and Nutritional Diseases, Ankara; ${ }^{4}$ Ankara University, Faculty of Agriculture, Department of Soil Science and Plant Nutrition, Ankara; ${ }^{5}$ TOBB University of Economics and Technology, Department of Mechanical Engineering, Ankara, Turkey.

aORCID: 0000-0002-4117-1098; bORCID: 0000-0002-1321-0280; ' $\mathrm{ORCID}$ : 0000-0001-8868-5291; ${ }^{\mathrm{d} O R C I D: 0000-0002-7997-9412 ;}{ }^{\mathrm{e} O R C I D: 0000-0001-6352-8302}$
\end{abstract}

Corresponding author: fatmakubra.erbay@gmail.com

Received date: 17.02.2020 - Accepted date: 09.07.2020

\begin{abstract}
Bone problems are highly prevalent in laying hens. These problems affect the welfare, production and economic losses. Bone development begins in the embryonic period, and if the skeletal system develops well at that time, the subsequent production period can be affected positively. The present experiment aimed to investigate the effect of genotype on biomechanical parameters and composition of bone in the laying hen embryos. For this purpose, 360 fertilized eggs were obtained from two brown (Atak-S and Brown Nick) and two white (Atabey and Nick) layer breeders and incubated. Metatarsus, tibia and femur properties were examined on the embryonic d 19 and 21. Results showed that genotype played an important role in determining the biomechanical properties and mineral composition of the metatarsus, tibia and femur in the embryonic period. Examined bone characteristics improved with embryonic age. The least mineralization was observed in the metatarsus bone. In conclusion, bone properties were infleunced from the genotype. However, these differences were not related with laying hens being white or brown. The effect of the interaction between genotype and embryonic age on the bone properties should be considered.
\end{abstract}

Keywords: Bone, embryo, genotype, laying hen,

\section{Yumurtacı tavuk embriyolarında genotipin kemik biyomekanik özellikleri ve bileşimine etkileri}

Özet: Yumurtacı tavuklarda kemik sorunları oldukça yaygındır. Bu sorunlar refahı, üretimi ve ekonomik kayıpları etkiler. Kemik gelişimi embriyonik dönemde başladığından bu dönemde iskelet sistemi iyi gelişirse, sonraki üretim periyodu olumlu yönde etkilenebilir. Bu çalışmada genotipin yumurtacı tavuk embriyolarında kemiğin biyomekanik özellikleri ve bileşimine etkisini incelemek amaçlanmıştır. Bu amaçla iki kahverengi (Atak-S ve Kahverengi Nick) ve iki beyaz (Atabey ve Nick) yumurtacı damızlıklardan elde edilen 360 adet döllü yumurta toplanmış ve inkübe edilmiştir. Embiyonik dönemin 19 ve 21. günlerinde metatarsus, tibia ve femur özellikleri incelenmiştir. Sonuçlar, embriyonik dönemde genotipin metatarsus, tibia ve femurun biyomekanik özellikleri ve mineral bileşiminde önemli bir rol oynadığını göstermiştir. Embriyonik yaşın artmasıyla incelenen kemik özellikleri iyileşmiştir. En az minerilizasyon metatarsus kemiğinde gözlenmiştir. Sonuç olarak, kemik özellikleri genotipten etkilenmiştir. Fakat bu farklılıklar beyaz veya kahverengi yumurtacı tavuk olmasıyla ilişkili değildir. Genotip ve embriyonik yaş arasındaki etkileşimin kemik özellikleri üzerindeki etkisi dikkate alınmalıdır.

Anahtar sözcükler: Embriyo, genotip, kemik, yumurtacı tavuk

\section{Introduction}

Laying hens have a skeleton to provide mobility support, protection and store for essential minerals especially calcium. Welfare, health and performance of hen and economic implications are related to skeletal development (10). The skeleton of birds is composed of a mineral part (70\%), organic part (20\%) and water (10\%). Most of the bones' mineral structure is composed of calcium and phosphorus $(10,22)$. In modern table egg production, companies use different brown and white laying hybrids depending on breeding methods. There are significant differences between white and brown laying 
hybrids in terms of body weight, feed consumption, egg weight and egg production $(14,15)$. However, genetic selection for higher production rate has come with unintended consequences; in particular, bone problems, depending on the rearing system, diet and hen's age. By the end of egg production the hens are susceptible to osteoporosis $(11,23)$. Bone fractures caused by osteoporosis are rated as serious welfare problems (18) resulting in increased economic losses through increased mortality and decreased eggshell quality. The skeletal problems involve mainly leg problems (25).

In fact, the basis of bone development takes place in the incubation. Femur and tibia start developing at 3.5 days of incubation, however, calcification begins at $10^{\text {th }}$ day of incubation (17). Around the $10^{\text {th }}$ day of incubation, calcium from the eggshell is transported to the embryo via chorioallantoic membrane (9). Bone calcium content also increases sharply from day 14 of incubation and starts to plateau at day 19 of incubation (12). The last phase of embryo development is marked by dramatic physiological and metabolic changes (13). Only few studies are available investigating the differences in bone development in the embryonic period of the different layer hybrids. Well-formed skeleton within the egg might increase the chick's healthiness in the layer period. Therefore, the aim of this study was to examine the differences regarding development and properties of leg bones during the embryonic age in different brown and white layer hybrids.

\section{Material and Methods}

The animal experimental protocol was approved by the Ankara University Animal Care and Use Committee (2015/5/102). When determining the sample to be used in the study the Power of test (1- $\beta$ ) was 0.80 calculated by G. Power statistical packet software. A total of 360 fertilized eggs were obtained from Atak-S, Atabey, Brown Nick and Nick layer breeders at 28 weeks of age. All eggs were numbered and weighed. Eggs were loaded to the incubator (Çimuka Incubator, Ankara, Turkey) set to $37.7^{\circ} \mathrm{C}$ and $53 \% \mathrm{RH}$ with $45^{\circ}$ rotation every hour. On $\mathrm{d}$ 18 , the eggs were transferred to the hatcher (Çimuka Incubator, Ankara, Turkey) set to $37.5^{\circ} \mathrm{C}$ and $70 \% \mathrm{RH}$. Eggs were placed in individual boxes in the hatcher allowing specific identification of each hatched chick on d 21 (15).

At the beginning of 19 and $21 \mathrm{~d}$ of incubation, twelve eggs from each genotype were randomly selected, weighed and opened. Embryos were sacrificed by cervical dislocation. Metatarsus, tibia and femur bones were dissected and cleared of all soft tissues. Bones were stored at $-20^{\circ} \mathrm{C}$ until analysis and dissolved at room temperature just before testing. Each bone was weighed using an analytical scale to the nearest $0.01 \mathrm{mg}$. Length was determined from the proximal end to distal end, and the width at the medial diaphysis (1). Leg bones from each embryo were subjected to the three-point bending test until failure occurred. Test was performed on Instron 5944 testing frame (Instron, Norwood, MA, USA). Loading rate was $5 \mathrm{~mm} / \mathrm{min}$. Span length was $10 \mathrm{~mm}$ for tibias. In bending test, span length should be about 16 times the thickness of the specimen generally. However, due to the nonuniform structure of bone, length-to-width ratio of 16:1 cannot be achieved. Therefore, $10 \mathrm{~mm}$ is the maximum span length which we can measure flexure of the bone. Load was applied to the midpoint of the shaft. Load vs displacement data was collected for each sample. Stiffness values were calculated from the slope of linear region of the load displacement curves. Breaking force was determined from the load displacement curves as well. Breaking force was defined as load at failure. Yield load is the load where permanent deformation of the system begins. Displacement at yield load is the displacement at which permanent deformation begins.

Dry matter and ash in the bones were determined according to the AOAC (3) methods. For the determination of mineral levels $(\mathrm{Zn}, \mathrm{Cu}, \mathrm{Mn}, \mathrm{Fe}, \mathrm{Na}, \mathrm{K}$, $\mathrm{Ca}, \mathrm{P}$ and $\mathrm{Mg}$ ), bones were analyzed (5) using an ICP-OES (Perkin Elmer Optima $^{\mathrm{TM}}$ DV 2100 Model, Dual View, Perkin Elmer Life and Analytical Sciences, Shelton, CT, USA). The values of these minerals were shown as per $\mathrm{kg}$ DM.

Statistical analyses: All of the experimental results are presented as mean \pm SEM. Two-way ANOVA was used for all data in order to test for main and interactive effects of genotype and embryonic age. If ANOVA revealed significant effects, it was followed by Tukey test. Statistical significance was accepted at $\mathrm{P} \leq 0.05$ (6).

\section{Results}

All geometrical and biomechanical parameters of metatarsus measured were affected by genotype except displacement at yield (Table 1). The major difference among genotype groups was observed in Nick embryos. Metatarsus of Nick embryos was found to be the heaviest and the longest comparing with other genotypes $(\mathrm{P}<0.001)$. Width, breaking force, stiffness and yield load of metatarsus in the Nick and Brown Nick embryos were found higher than those of the metatarsus in the Atabey and Atak-S embryos. As the embryonic age progressed, metatarsus properties were also increased except displacement at yield $(\mathrm{P}<0.001)$. Two way interactions between hybrid and embryonic age were found in the weight, stiffness and yield load of metatarsus $(\mathrm{P}<0.01)$. Because, the increases in the weight, stiffness and yield load of metatarsus in Nick embryos were the highest from embryonic d (E) 19 to E21 among the other embryos. 
Table 1. Effect of genotype and embryonic age on geometrical and biomechanical parameters in the metatarsus

\begin{tabular}{|c|c|c|c|c|c|c|c|c|}
\hline Genotype & $\begin{array}{c}\text { Embryonic } \\
\text { age }\end{array}$ & $\begin{array}{c}\text { Weight } \\
\text { (g) }\end{array}$ & $\begin{array}{c}\begin{array}{c}\text { Length } \\
(\mathrm{mm})\end{array} \\
\end{array}$ & $\begin{array}{l}\text { Width } \\
(\mathbf{m m})\end{array}$ & $\begin{array}{l}\text { Breaking force } \\
(\mathbf{N})\end{array}$ & $\begin{array}{c}\text { Stiffness } \\
(\mathrm{N} / \mathrm{mm})\end{array}$ & $\begin{array}{c}\text { Yield load } \\
(\mathrm{N})\end{array}$ & $\begin{array}{l}\text { Displacement } \\
\text { at yield (mm) }\end{array}$ \\
\hline \multicolumn{9}{|l|}{ White layer } \\
\hline Atabey & & $0.16^{\mathrm{c}}$ & $19.95^{\mathrm{ab}}$ & $1.38^{\mathrm{b}}$ & $4.73^{b}$ & $5.68^{\mathrm{b}}$ & $3.00^{\mathrm{b}}$ & 0.87 \\
\hline Nick & & $0.22^{\mathrm{a}}$ & $20.72^{\mathrm{a}}$ & $1.55^{\mathrm{a}}$ & $6.30^{\mathrm{a}}$ & $9.83^{\mathrm{a}}$ & $4.52^{\mathrm{a}}$ & 0.78 \\
\hline \multicolumn{9}{|l|}{ Brown layer } \\
\hline Atak-S & & $0.15^{\mathrm{c}}$ & $18.67^{\mathrm{c}}$ & $1.32^{\mathrm{b}}$ & $4.82^{b}$ & $7.08^{\mathrm{b}}$ & $2.72^{\mathrm{b}}$ & 0.73 \\
\hline \multirow[t]{3}{*}{ Brown Nick } & & $0.19^{\mathrm{b}}$ & $19.65^{\mathrm{b}}$ & $1.54^{\mathrm{a}}$ & $5.90^{\mathrm{a}}$ & $9.31^{\mathrm{a}}$ & $4.07^{\mathrm{a}}$ & 0.81 \\
\hline & E19 & 0.15 & 19.22 & 1.38 & 4.83 & 6.17 & 2.95 & 0.85 \\
\hline & E21 & 0.21 & 20.28 & 1.52 & 6.04 & 9.78 & 4.21 & 0.75 \\
\hline \multirow[t]{2}{*}{ Atabey } & E19 & 0.14 & 19.49 & 1.34 & 4.01 & 3.93 & 2.51 & 0.88 \\
\hline & E21 & 0.19 & 20.41 & 1.42 & 5.46 & 7.42 & 3.48 & 0.86 \\
\hline \multirow[t]{2}{*}{ Nick } & E19 & 0.18 & 20.26 & 1.48 & 5.27 & 6.78 & 3.08 & 0.83 \\
\hline & E21 & 0.26 & 21.18 & 1.63 & 7.33 & 12.88 & 5.96 & 0.73 \\
\hline \multirow[t]{2}{*}{ Atak-S } & E19 & 0.12 & 17.88 & 1.18 & 4.56 & 6.48 & 2.87 & 0.76 \\
\hline & E21 & 0.18 & 19.45 & 1.45 & 5.08 & 7.68 & 2.58 & 0.71 \\
\hline \multirow[t]{2}{*}{ Brown Nick } & E19 & 0.19 & 19.23 & 1.51 & 5.49 & 7.48 & 3.33 & 0.92 \\
\hline & $\mathrm{E} 21$ & 0.20 & 20.06 & 1.58 & 6.31 & 11.15 & 4.81 & 0.70 \\
\hline \multirow{2}{*}{\multicolumn{2}{|c|}{ SEM }} & 0.003 & 0.108 & 0.020 & 0.105 & 0.222 & 0.132 & 0.031 \\
\hline & & & & & $\mathrm{P}$-value & & & \\
\hline \multicolumn{2}{|c|}{ Genotype } & $<0.001$ & $<0.001$ & $<0.001$ & $<0.001$ & $<0.001$ & $<0.001$ & 0.113 \\
\hline \multicolumn{2}{|c|}{ Embryonic age } & $<0.001$ & $<0.001$ & 0.001 & $<0.001$ & $<0.001$ & $<0.001$ & 0.430 \\
\hline \multicolumn{2}{|c|}{ Genotype X Embryonic age } & 0.003 & 0.628 & 0.313 & 0.061 & 0.004 & 0.001 & 0.652 \\
\hline
\end{tabular}

a,b,c Means within a column with different superscript letters differ.

The values of weight, length, breaking force and stiffness of tibia were found to be the highest in the Nick embryos $(\mathrm{P} \leq 0.001)$ as in the metatarsus (Table 2$)$. Width of tibia values were found as $1.48,1.55,1.53$ and $1.60 \mathrm{~mm}$ in the Atabey, Nick, Atak-S and Brown Nick embryos, respectively. Brown Nick had the widest tibia in the embryonic period $(\mathrm{P}<0.01)$. Yield loads were 4.29, 5.14, 3.74 and $5.44 \mathrm{~N}$ for Atabey, Nick, Atak-S and Brown Nick embryos, respectively; tibia of Nick and Brown Nick embryos had higher yield load values than the other examined embryos $(\mathrm{P}<0.001)$. Displacement at yield was not found different in the genotype groups. Weight ( $\mathrm{P}<0.001)$, length $(\mathrm{P}<0.001)$, width $(\mathrm{P}<0.05)$, breaking force $(\mathrm{P}<0.001)$, stiffness $(\mathrm{P}<0.001)$ and yield load $(\mathrm{P}<0.001)$ increased with the increase in the embryonic age from E19 to E21. But, displacement at yield did not differ between embryonic age groups. Interaction was found only for length and yield load of tibia $(\mathrm{P}<0.05)$.

Nick embryos had the heaviest $(\mathrm{P}<0.01)$ and the longest $(\mathrm{P}<0.001)$ femur than the others in the examined embryonic period (Table 3). Femur widths were found as $1.48,1.57,1.43$ and $1.58 \mathrm{~mm}$ in the Atabey, Nick, Atak-S and Brown Nick embryos, respectively. The lowest breaking force value was found in the Atak-S embryos. Stiffness values were 11.10, 14.38, 9.86 and $12.52 \mathrm{~N} / \mathrm{mm}$ in the Atabey, Nick, Atak-S and Brown Nick embryos, respectively. The highest stiffness $(\mathrm{P}<0.001)$ and yield load $(\mathrm{P}<0.05)$ of femur were found in the Nick embryos. Displacement at yield did not differ among the genotype groups. Examined geometrical and biomechanical parameters of femur except of displacement at yield increased from E19 to E21 $(\mathrm{P}<0.01)$. Increase in the femur stiffness from E19 to E21 was lowest in the Atabey embryos, and this situation caused the genotype and embryonic age interaction.

Mineral levels of metatarsus, tibia and femur are presented in Table 4, 5 and 6. $\mathrm{Fe}, \mathrm{Zn}, \mathrm{Na}$ and $\mathrm{Ca}$ levels in the metatarsus; ash, $\mathrm{Fe}, \mathrm{Zn}, \mathrm{Na}$ and $\mathrm{Ca}$ levels in the tibia, and ash, $\mathrm{Fe}, \mathrm{Zn}, \mathrm{Na}$ and $\mathrm{Ca}$ levels in the femur were affected by the genotype $(\mathrm{P}<0.05)$. All examined minerals in the metatarsus, tibia and femur except $\mathrm{Mg}$ in the metatarsus and tibia increased with embryonic age $(\mathrm{P} \leq 0.05)$. The highest $\mathrm{Fe}$ content in the metatarsus, tibia and femur was found in the Nick and Brown Nick embryos $(\mathrm{P}<0.001)$. The highest $\mathrm{Ca}$ level was found as 223.49 $(\mathrm{P}<0.01), 272.85 \quad(\mathrm{P}<0.001)$ and $276.34 \mathrm{~g} / \mathrm{kg} \quad \mathrm{DM}$ $(\mathrm{P}<0.001)$ in the metatarsus, tibia and femur bones, respectively in the Brown Nick embryos.

In metatarsus, interaction effects between genotype and embryonic age were observed for $\mathrm{Fe}$ and $\mathrm{Na}$ concentrations. This was due to the higher increment in $\mathrm{Fe}$ level in the Brown Nick embryos and lower increase in $\mathrm{Na}$ level in the Atabey embryos from E19 to E21. In the tibia bone, increase in the ash, $\mathrm{Cu}, \mathrm{Fe}$ and $\mathrm{P}$ concentrations from E19 to E21 varied by genotype and this resulted in interaction. Same trend was also observed for ash, $\mathrm{Cu}, \mathrm{Fe}$, $\mathrm{Na}$ and P levels in the femur from E19 to E21. 
Table 2. Effect of genotype and embryonic age on geometrical and biomechanical parameters in the tibia

\begin{tabular}{|c|c|c|c|c|c|c|c|c|}
\hline Genotype & $\begin{array}{c}\text { Embryonic } \\
\text { age }\end{array}$ & $\begin{array}{l}\text { Weight } \\
\text { (g) }\end{array}$ & $\begin{array}{c}\text { Length } \\
\text { (mm) }\end{array}$ & $\begin{array}{l}\text { Width } \\
\text { (mm) }\end{array}$ & $\begin{array}{l}\text { Breaking force } \\
\text { (N) }\end{array}$ & $\begin{array}{l}\text { Stiffness } \\
(\mathbf{N} / \mathbf{m m})\end{array}$ & $\begin{array}{l}\text { Yield load } \\
\text { (N) }\end{array}$ & $\begin{array}{l}\text { Displacement } \\
\text { at yield }(\mathrm{mm})\end{array}$ \\
\hline \multicolumn{9}{|l|}{ White layer } \\
\hline Atabey & & $0.23^{\mathrm{bc}}$ & $27.06^{\mathrm{b}}$ & $1.48^{\mathrm{b}}$ & $7.03^{\mathrm{b}}$ & $16.16^{\mathrm{bc}}$ & $4.29^{\mathrm{b}}$ & 0.45 \\
\hline Nick & & $0.27^{\mathrm{a}}$ & $28.53^{\mathrm{a}}$ & $1.55^{\mathrm{ab}}$ & $7.71^{\mathrm{a}}$ & $20.52^{\mathrm{a}}$ & $5.14^{\mathrm{a}}$ & 0.42 \\
\hline \multicolumn{9}{|l|}{ Brown layer } \\
\hline Atak-S & & $0.21^{\mathrm{c}}$ & $26.28^{\mathrm{b}}$ & $1.53^{\mathrm{ab}}$ & $6.22^{\mathrm{c}}$ & $14.80^{c}$ & $3.74^{\mathrm{b}}$ & 0.63 \\
\hline \multirow[t]{3}{*}{ Brown Nick } & & $0.26^{\mathrm{ab}}$ & $27.06^{\mathrm{b}}$ & $1.60^{\mathrm{a}}$ & $7.50^{\mathrm{ab}}$ & $18.27^{\mathrm{ab}}$ & $5.44^{\mathrm{a}}$ & 0.44 \\
\hline & E19 & 0.20 & 26.20 & 1.51 & 6.36 & 14.42 & 4.03 & 0.45 \\
\hline & E21 & 0.28 & 28.27 & 1.57 & 7.87 & 20.32 & 5.27 & 0.52 \\
\hline \multirow[t]{2}{*}{ Atabey } & E19 & 0.18 & 25.81 & 1.44 & 6.06 & 13.77 & 3.80 & 0.40 \\
\hline & E21 & 0.28 & 28.30 & 1.53 & 8.00 & 18.55 & 4.77 & 0.50 \\
\hline \multirow[t]{2}{*}{ Nick } & E19 & 0.23 & 28.02 & 1.54 & 6.74 & 15.18 & 3.73 & 0.48 \\
\hline & E21 & 0.31 & 29.04 & 1.55 & 8.68 & 25.33 & 6.54 & 0.36 \\
\hline \multirow[t]{2}{*}{ Atak-S } & E19 & 0.17 & 24.78 & 1.48 & 5.62 & 13.24 & 3.65 & 0.49 \\
\hline & E21 & 0.26 & 27.79 & 1.58 & 6.82 & 16.35 & 3.83 & 0.78 \\
\hline \multirow[t]{2}{*}{ Brown Nick } & E19 & 0.22 & 26.19 & 1.58 & 7.02 & 15.50 & 4.94 & 0.42 \\
\hline & E21 & 0.29 & 27.93 & 1.62 & 7.98 & 21.04 & 5.94 & 0.46 \\
\hline \multicolumn{2}{|c|}{ SEM } & 0.004 & 0.127 & 0.012 & 0.092 & 0.491 & 0.125 & 0.051 \\
\hline \multicolumn{9}{|c|}{ P-value } \\
\hline \multicolumn{2}{|c|}{ Genotype } & $<0.001$ & $<0.001$ & 0.007 & $<0.001$ & 0.001 & $<0.001$ & 0.410 \\
\hline \multicolumn{2}{|c|}{ Embryonic age } & $<0.001$ & $<0.001$ & 0.012 & $<0.001$ & $<0.001$ & $<0.001$ & 0.453 \\
\hline \multicolumn{2}{|c|}{ Genotype X Embryonic age } & 0.471 & 0.036 & 0.467 & 0.137 & 0.079 & 0.003 & 0.549 \\
\hline
\end{tabular}

a,b,c Means within a column with different superscript letters differ.

Table 3. Effect of genotype and embryonic age on geometrical and biomechanical parameters in the femur

\begin{tabular}{|c|c|c|c|c|c|c|c|c|}
\hline Genotype & $\begin{array}{c}\text { Embryonic } \\
\text { age }\end{array}$ & $\begin{array}{c}\text { Weight } \\
\text { (g) }\end{array}$ & $\begin{array}{c}\text { Length } \\
\text { (mm) }\end{array}$ & $\begin{array}{l}\text { Width } \\
\text { (mm) }\end{array}$ & $\begin{array}{l}\text { Breaking force } \\
\text { (N) }\end{array}$ & $\begin{array}{l}\text { Stiffness } \\
(\mathrm{N} / \mathrm{mm})\end{array}$ & $\begin{array}{l}\text { Yield load } \\
(\mathrm{N})\end{array}$ & $\begin{array}{l}\text { Displacement } \\
\text { at yield (mm) }\end{array}$ \\
\hline \multicolumn{9}{|l|}{ White layer } \\
\hline Atabey & & $0.15^{\mathrm{b}}$ & $19.22^{\mathrm{b}}$ & $1.48^{\mathrm{bc}}$ & $6.49^{a}$ & $11.10^{\mathrm{b}}$ & $4.54^{\mathrm{ab}}$ & 0.72 \\
\hline Nick & & $0.18^{\mathrm{a}}$ & $20.40^{\mathrm{a}}$ & $1.57^{\mathrm{ab}}$ & $7.31^{\mathrm{a}}$ & $14.38^{\mathrm{a}}$ & $4.90^{\mathrm{a}}$ & 0.68 \\
\hline \multicolumn{9}{|l|}{ Brown layer } \\
\hline Atak-S & & $0.13^{\mathrm{b}}$ & $18.44^{\mathrm{b}}$ & $1.43^{\mathrm{c}}$ & $5.34^{\mathrm{b}}$ & $9.86^{\mathrm{b}}$ & $3.32^{\mathrm{b}}$ & 0.62 \\
\hline \multirow[t]{3}{*}{ Brown Nick } & & $0.15^{\mathrm{b}}$ & $19.18^{\mathrm{b}}$ & $1.58^{\mathrm{a}}$ & $6.48^{\mathrm{a}}$ & $12.52^{\mathrm{ab}}$ & $4.36^{\mathrm{ab}}$ & 0.74 \\
\hline & E19 & 0.14 & 18.75 & 1.47 & 5.61 & 9.21 & 3.31 & 0.70 \\
\hline & E21 & 0.17 & 19.87 & 1.55 & 7.21 & 14.72 & 5.25 & 0.68 \\
\hline \multirow[t]{2}{*}{ Atabey } & E19 & 0.13 & 18.87 & 1.44 & 5.91 & 10.53 & 3.71 & 0.65 \\
\hline & E21 & 0.17 & 19.57 & 1.51 & 7.06 & 11.66 & 5.37 & 0.80 \\
\hline \multirow[t]{2}{*}{ Nick } & E19 & 0.16 & 19.82 & 1.49 & 6.49 & 10.61 & 4.20 & 0.75 \\
\hline & E21 & 0.20 & 20.97 & 1.65 & 8.14 & 18.14 & 5.50 & 0.60 \\
\hline \multirow[t]{2}{*}{ Atak-S } & E19 & 0.12 & 17.65 & 1.42 & 4.53 & 6.78 & 2.08 & 0.69 \\
\hline & E21 & 0.15 & 19.23 & 1.44 & 6.16 & 12.93 & 4.56 & 0.55 \\
\hline \multirow[t]{2}{*}{ Brown Nick } & E19 & 0.14 & 18.67 & 1.54 & 5.50 & 8.90 & 3.23 & 0.71 \\
\hline & E21 & 0.16 & 19.69 & 1.61 & 7.46 & 16.14 & 5.50 & 0.77 \\
\hline \multirow[t]{2}{*}{ SEM } & & 0.004 & 0.114 & 0.013 & 0.113 & 0.363 & 0.186 & 0.029 \\
\hline & \multicolumn{8}{|c|}{ P-value } \\
\hline \multicolumn{2}{|c|}{ Genotype } & 0.002 & $<0.001$ & $<0.001$ & $<0.001$ & $<0.001$ & 0.024 & 0.491 \\
\hline \multicolumn{2}{|c|}{ Embryonic age } & 0.001 & $<0.001$ & 0.002 & $<0.001$ & $<0.001$ & $<0.001$ & 0.749 \\
\hline \multicolumn{2}{|c|}{ Genotype X Embryonic age } & 0.698 & 0.630 & 0.249 & 0.671 & 0.015 & 0.692 & 0.212 \\
\hline
\end{tabular}

$\overline{\mathrm{a}, \mathrm{b}, \mathrm{c}}$ Means within a column with different superscript letters differ. 
Table 4. Effect of genotype and embryonic age on metatarsus composition

\begin{tabular}{|c|c|c|c|c|c|c|c|c|c|c|c|}
\hline Genotype & $\begin{array}{c}\text { Embryonic } \\
\text { age }\end{array}$ & $\begin{array}{c}\text { Ash } \\
\text { (g/100g } \\
\text { DM) }\end{array}$ & $\begin{array}{c}\mathrm{Cu} \\
(\mathrm{mg} / \mathrm{kg} \\
\mathrm{DM})\end{array}$ & $\begin{array}{c}\mathrm{Fe} \\
(\mathrm{mg} / \mathrm{kg} \\
\mathrm{DM})\end{array}$ & $\begin{array}{c}\mathrm{Mn} \\
(\mathrm{mg} / \mathrm{kg} \\
\mathrm{DM})\end{array}$ & $\begin{array}{c}\mathrm{Zn} \\
(\mathrm{mg} / \mathrm{kg} \\
\mathrm{DM})\end{array}$ & $\begin{array}{l}\mathrm{Na} \\
(\mathrm{g} / \mathrm{kg} \\
\mathrm{DM})\end{array}$ & $\begin{array}{l}\mathrm{Ca} \\
(\mathrm{g} / \mathrm{kg} \\
\mathrm{DM})\end{array}$ & $\begin{array}{c}P \\
(\mathrm{~g} / \mathrm{kg} \\
\mathrm{DM})\end{array}$ & $\begin{array}{l}\text { Mg } \\
\text { (g/kg } \\
\text { DM) }\end{array}$ & $\begin{array}{c}\text { K } \\
(\mathrm{g} / \mathrm{kg} \\
\mathrm{DM})\end{array}$ \\
\hline \multicolumn{12}{|l|}{ White layer } \\
\hline Atabey & & 40.87 & 0.33 & $163.23^{\mathrm{c}}$ & 0.49 & $221.87^{\mathrm{a}}$ & $2.71^{\mathrm{b}}$ & $206.51^{\mathrm{ab}}$ & 106.43 & 2.78 & 2.67 \\
\hline Nick & & 42.89 & 0.36 & $218.42^{\mathrm{a}}$ & 0.52 & $201.59^{\mathrm{a}}$ & $3.36^{\mathrm{a}}$ & $210.86^{\mathrm{ab}}$ & 105.01 & 3.35 & 2.83 \\
\hline \multicolumn{12}{|l|}{ Brown layer } \\
\hline Atak-S & & 43.88 & 0.43 & $201.66^{\mathrm{b}}$ & 0.54 & $181.63^{b}$ & $3.72^{\mathrm{a}}$ & $201.40^{\mathrm{b}}$ & 106.88 & 2.76 & 2.65 \\
\hline \multirow[t]{3}{*}{ Brown Nick } & & 41.78 & 0.46 & $256.29^{\mathrm{a}}$ & 0.58 & $207.59^{\mathrm{a}}$ & $3.40^{\mathrm{a}}$ & $223.49^{a}$ & 113.21 & 3.23 & 2.45 \\
\hline & E19 & 40.21 & 0.33 & 165.53 & 0.45 & 147.19 & 2.71 & 198.70 & 95.89 & 2.95 & 2.14 \\
\hline & $\mathrm{E} 21$ & 44.51 & 0.46 & 254.27 & 0.61 & 259.15 & 3.88 & 222.44 & 119.87 & 3.37 & 3.16 \\
\hline \multirow[t]{2}{*}{ Atabey } & E19 & 39.60 & 0.22 & 141.13 & 0.41 & 151.77 & 2.65 & 192.52 & 94.03 & 2.72 & 2.16 \\
\hline & E21 & 42.15 & 0.45 & 185.32 & 0.56 & 291.95 & 2.77 & 220.49 & 118.83 & 2.84 & 3.18 \\
\hline \multirow[t]{2}{*}{ Nick } & E19 & 39.60 & 0.29 & 165.06 & 0.41 & 145.75 & 2.57 & 199.69 & 92.13 & 3.25 & 2.33 \\
\hline & E21 & 46.18 & 0.43 & 271.78 & 0.64 & 257.43 & 4.15 & 216.33 & 117.88 & 3.45 & 3.34 \\
\hline \multirow[t]{2}{*}{ Atak-S } & E19 & 42.36 & 0.39 & 166.29 & 0.45 & 134.61 & 3.08 & 186.48 & 99.06 & 3.07 & 2.30 \\
\hline & E21 & 45.40 & 0.46 & 237.03 & 0.63 & 228.66 & 4.35 & 216.33 & 114.70 & 3.45 & 3.00 \\
\hline \multirow[t]{2}{*}{ Brown Nick } & E19 & 39.27 & 0.41 & 189.65 & 0.55 & 156.63 & 2.55 & 216.10 & 98.34 & 2.76 & 1.77 \\
\hline & E21 & 44.29 & 0.50 & 322.92 & 0.61 & 258.54 & 4.25 & 230.89 & 128.09 & 3.69 & 3.13 \\
\hline \multirow[t]{2}{*}{ SEM } & & 0.426 & 0.017 & 4.675 & 0.036 & 4.996 & 0.102 & 2.306 & 1.311 & 0.116 & 0.112 \\
\hline & & & \multicolumn{9}{|c|}{ P-value } \\
\hline \multicolumn{2}{|c|}{ Genotype } & 0.074 & 0.087 & $<0.001$ & 0.837 & 0.041 & 0.008 & 0.006 & 0.124 & 0.319 & 0.692 \\
\hline \multicolumn{2}{|c|}{ Embryonic age } & $<0.001$ & $<0.001$ & $<0.001$ & 0.034 & $<0.001$ & $<0.001$ & $<0.001$ & $<0.001$ & 0.075 & $<0.001$ \\
\hline \multicolumn{2}{|c|}{ Genotype X Embryonic age } & 0.340 & 0.328 & 0.006 & 0.883 & 0.390 & 0.036 & 0.637 & 0.253 & 0.599 & 0.774 \\
\hline
\end{tabular}

a,b,c Means within a column with different superscript letters differ.

Table 5. Effect of genotype and embryonic age on tibia composition

\begin{tabular}{|c|c|c|c|c|c|c|c|c|c|c|c|}
\hline Genotype & $\begin{array}{c}\text { Embryonic } \\
\text { age }\end{array}$ & $\begin{array}{c}\text { Ash } \\
(\mathrm{g} / 100 \mathrm{~g} \\
\text { DM) }\end{array}$ & $\begin{array}{c}\mathrm{Cu} \\
(\mathrm{mg} / \mathrm{kg} \\
\mathrm{DM}) \\
\end{array}$ & $\begin{array}{c}\mathrm{Fe} \\
(\mathrm{mg} / \mathrm{kg} \\
\mathrm{DM})\end{array}$ & $\begin{array}{c}\mathrm{Mn} \\
(\mathrm{mg} / \mathrm{kg} \\
\mathrm{DM})\end{array}$ & $\begin{array}{c}\mathrm{Zn} \\
(\mathrm{mg} / \mathrm{kg} \\
\mathrm{DM})\end{array}$ & $\begin{array}{l}\mathrm{Na} \\
(\mathrm{g} / \mathrm{kg} \\
\mathrm{DM}) \\
\end{array}$ & $\begin{array}{c}\text { Ca } \\
(\mathrm{g} / \mathrm{kg} \\
\mathrm{DM}) \\
\end{array}$ & $\begin{array}{c}\begin{array}{c}P \\
(g / k g\end{array} \\
\text { DM) }\end{array}$ & $\begin{array}{c}\text { Mg } \\
\text { (g/kg } \\
\text { DM) } \\
\end{array}$ & $\begin{array}{c}\text { K } \\
\text { (g/kg } \\
\text { DM) } \\
\end{array}$ \\
\hline \multicolumn{12}{|l|}{ White layer } \\
\hline Atabey & & $50.03^{\mathrm{bc}}$ & 0.58 & $215.84^{\mathrm{c}}$ & 0.64 & $265.50^{\mathrm{a}}$ & $3.16^{\mathrm{b}}$ & $244.15^{\mathrm{b}}$ & 120.66 & 3.12 & 3.03 \\
\hline Nick & & $52.41^{\mathrm{a}}$ & 0.54 & $280.13^{\mathrm{a}}$ & 0.60 & $244.14^{\mathrm{a}}$ & $4.08^{\mathrm{a}}$ & $246.41^{b}$ & 115.96 & 3.72 & 3.14 \\
\hline \multicolumn{12}{|l|}{ Brown layer } \\
\hline Atak-S & & $51.34^{\mathrm{ab}}$ & 0.63 & $256.27^{b}$ & 0.65 & $221.32^{b}$ & $4.40^{\mathrm{a}}$ & $247.27^{\mathrm{b}}$ & 114.45 & 3.52 & 2.95 \\
\hline \multirow[t]{3}{*}{ Brown Nick } & & $48.17^{\mathrm{c}}$ & 0.63 & $307.12^{\mathrm{a}}$ & 0.77 & $271.77^{\mathrm{a}}$ & $3.82^{\mathrm{ab}}$ & $272.85^{\mathrm{a}}$ & 121.49 & 3.51 & 2.69 \\
\hline & E19 & 48.74 & 0.52 & 205.44 & 0.54 & 185.67 & 3.19 & 232.37 & 103.38 & 3.25 & 2.36 \\
\hline & E21 & 52.24 & 0.67 & 324.23 & 0.79 & 315.69 & 4.54 & 272.97 & 132.90 & 3.69 & 3.54 \\
\hline \multirow[t]{2}{*}{ Atabey } & E19 & 49.78 & 0.39 & 178.36 & 0.53 & 180.79 & 2.99 & 225.19 & 101.15 & 2.99 & 2.47 \\
\hline & E21 & 50.29 & 0.76 & 253.31 & 0.76 & 350.21 & 3.34 & 263.11 & 140.17 & 3.24 & 3.59 \\
\hline \multirow[t]{2}{*}{ Nick } & E19 & 51.37 & 0.46 & 209.81 & 0.45 & 182.76 & 3.29 & 222.10 & 103.35 & 3.62 & 2.53 \\
\hline & E21 & 53.45 & 0.61 & 350.44 & 0.75 & 305.52 & 4.87 & 270.72 & 128.58 & 3.82 & 3.76 \\
\hline \multirow[t]{2}{*}{ Atak-S } & E19 & 48.56 & 0.62 & 208.65 & 0.56 & 160.65 & 3.54 & 224.92 & 104.22 & 3.33 & 2.48 \\
\hline & E21 & 54.12 & 0.65 & 303.89 & 0.73 & 282.00 & 5.26 & 269.61 & 124.68 & 3.72 & 3.46 \\
\hline \multirow[t]{2}{*}{ Brown Nick } & E19 & 45.23 & 0.61 & 224.95 & 0.63 & 218.49 & 2.93 & 257.27 & 104.80 & 3.06 & 1.98 \\
\hline & E21 & 51.12 & 0.66 & 389.28 & 0.91 & 325.05 & 4.70 & 288.43 & 138.18 & 3.96 & 3.39 \\
\hline \multirow[t]{2}{*}{ SEM } & & 0.327 & 0.021 & 4.364 & 0.054 & 5.148 & 0.123 & 1.915 & 1.211 & 0.123 & 0.122 \\
\hline & & & & \multicolumn{8}{|c|}{ P-value } \\
\hline \multicolumn{2}{|c|}{ Genotype } & $<0.001$ & 0.291 & $<0.001$ & 0.721 & 0.002 & 0.006 & $<0.001$ & 0.106 & 0.393 & 0.589 \\
\hline \multicolumn{2}{|c|}{ Embryonic age } & $<0.001$ & 0.001 & $<0.001$ & 0.028 & $<0.001$ & $<0.001$ & $<0.001$ & $<0.001$ & 0.082 & $<0.001$ \\
\hline \multicolumn{2}{|c|}{ Genotype X Embryonic age } & 0.009 & 0.021 & 0.002 & 0.976 & 0.167 & 0.144 & 0.392 & 0.038 & 0.734 & 0.920 \\
\hline
\end{tabular}


Table 6. Effect of genotype and embryonic age on femur composition

\begin{tabular}{|c|c|c|c|c|c|c|c|c|c|c|c|}
\hline Genotype & $\begin{array}{c}\text { Embryonic } \\
\text { age }\end{array}$ & $\begin{array}{c}\text { Ash } \\
\text { (g/100 } \\
\text { g DM) }\end{array}$ & $\begin{array}{c}\mathrm{Cu} \\
(\mathrm{mg} / \mathrm{kg} \\
\mathrm{DM})\end{array}$ & $\begin{array}{c}\mathrm{Fe} \\
(\mathrm{mg} / \mathrm{kg} \\
\mathrm{DM})\end{array}$ & $\begin{array}{c}\mathrm{Mn} \\
(\mathrm{mg} / \mathrm{kg} \\
\mathrm{DM})\end{array}$ & $\begin{array}{c}\text { Zn } \\
(\mathrm{mg} / \mathrm{kg} \\
\mathrm{DM})\end{array}$ & $\begin{array}{l}\mathrm{Na} \\
\text { (g/kg } \\
\mathrm{DM})\end{array}$ & $\begin{array}{c}\mathrm{Ca} \\
(\mathrm{g} / \mathrm{kg} \\
\mathrm{DM})\end{array}$ & $\begin{array}{c}P \\
\text { (g/kg } \\
\text { DM) }\end{array}$ & $\begin{array}{c}\mathrm{Mg} \\
(\mathrm{g} / \mathrm{kg} \\
\mathrm{DM})\end{array}$ & $\begin{array}{c}\mathrm{K} \\
(\mathrm{g} / \mathrm{kg} \\
\mathrm{DM})\end{array}$ \\
\hline \multicolumn{12}{|l|}{ White layer } \\
\hline Atabey & & $50.82^{\mathrm{a}}$ & 0.62 & $219.22^{b}$ & 0.68 & $268.60^{\mathrm{a}}$ & $3.31^{\mathrm{b}}$ & $249.15^{b}$ & 124.32 & 3.39 & 3.28 \\
\hline Nick & & $50.87^{\mathrm{a}}$ & 0.55 & $279.91^{\mathrm{a}}$ & 0.63 & $246.10^{\mathrm{a}}$ & $4.11^{\mathrm{a}}$ & $249.98^{b}$ & 117.75 & 3.94 & 3.29 \\
\hline \multicolumn{12}{|l|}{ Brown layer } \\
\hline Atak-S & & $52.30^{\mathrm{a}}$ & 0.66 & $256.86^{\mathrm{b}}$ & 0.66 & $226.28^{b}$ & $4.52^{\mathrm{a}}$ & $249.40^{\mathrm{b}}$ & 116.99 & 3.86 & 3.13 \\
\hline \multirow[t]{3}{*}{ Brown Nick } & & $48.89^{b}$ & 0.66 & $311.67^{a}$ & 0.78 & $273.20^{\mathrm{a}}$ & $4.14^{\mathrm{a}}$ & $276.34^{\mathrm{a}}$ & 124.18 & 3.71 & 2.88 \\
\hline & E19 & 48.57 & 0.54 & 209.13 & 0.59 & 188.95 & 3.46 & 237.72 & 106.31 & 3.41 & 2.64 \\
\hline & E21 & 52.86 & 0.71 & 324.70 & 0.79 & 318.14 & 4.57 & 274.72 & 135.31 & 4.01 & 3.65 \\
\hline \multirow[t]{2}{*}{ Atabey } & E19 & 50.77 & 0.43 & 186.78 & 0.55 & 185.75 & 3.32 & 233.55 & 106.15 & 3.07 & 2.87 \\
\hline & E21 & 50.86 & 0.81 & 251.66 & 0.81 & 351.46 & 3.30 & 264.76 & 142.49 & 3.61 & 3.68 \\
\hline \multirow[t]{2}{*}{ Nick } & E19 & 47.37 & 0.46 & 208.57 & 0.51 & 183.44 & 3.20 & 226.13 & 105.18 & 3.83 & 2.76 \\
\hline & E21 & 54.37 & 0.65 & 351.25 & 0.76 & 308.75 & 5.01 & 273.83 & 130.32 & 4.05 & 3.83 \\
\hline \multirow[t]{2}{*}{ Atak-S } & E19 & 50.00 & 0.64 & 210.80 & 0.63 & 166.20 & 3.97 & 227.35 & 107.41 & 3.59 & 2.78 \\
\hline & E21 & 54.59 & 0.69 & 302.91 & 0.69 & 286.36 & 3.30 & 271.44 & 126.56 & 4.12 & 3.49 \\
\hline \multirow[t]{2}{*}{ Brown Nick } & E19 & 46.14 & 0.64 & 230.36 & 0.66 & 220.40 & 3.36 & 263.85 & 106.50 & 3.16 & 2.14 \\
\hline & E21 & 51.63 & 0.68 & 392.98 & 0.90 & 326.00 & 4.91 & 288.84 & 141.86 & 4.26 & 3.61 \\
\hline \multirow[t]{2}{*}{ SEM } & & 0.313 & 0.022 & 4.375 & 0.052 & 5.199 & 0.099 & 1.790 & 1.265 & 0.117 & 0.115 \\
\hline & \multicolumn{11}{|c|}{ P-value } \\
\hline \multicolumn{2}{|c|}{ Genotype } & 0.002 & 0.278 & $<0.001$ & 0.758 & 0.005 & $<0.001$ & $<0.001$ & 0.065 & 0.298 & 0.550 \\
\hline \multicolumn{2}{|c|}{ Embryonic age } & $<0.001$ & $<0.001$ & $<0.001$ & 0.054 & $<0.001$ & $<0.001$ & $<0.001$ & $<0.001$ & 0.013 & $<0.001$ \\
\hline \multicolumn{2}{|c|}{ Genotype X Embryonic age } & 0.002 & 0.021 & $<0.001$ & 0.882 & 0.216 & 0.011 & 0.091 & 0.047 & 0.603 & 0.644 \\
\hline
\end{tabular}

${ }^{\mathrm{a}, \mathrm{b}}$ Means within a column with different superscript letters differ.

\section{Discussion and Conclusion}

For poultry, skeletal system is one of the most important development systems in the embryonic period. Genetic selection as a tool to obtain higher production has undesirable effects on the skeletal system resulting in major problems such as osteoporosis, fracture, paralysis and death (24). The better bone development in the embryonic period results in less bone problems during the production period. In the present study, clear differences were observed for geometrical and biomechanical parameters of leg bones obtained from different genotypes in the embryonic period. It is well documented that commercial brown hybrid layers represent the heavy weight type with stronger bones than the light weight type such as white layers (7). However, results in this study showed that this idea does not apply to the embryonic period. Because, almost all geometrical and biomechanical parameters of leg bones were found to be highest in the Nick embryos and second highest level was found in the Brown Nick embryos. Nick embryos had heavier and longer metatarsus and femur along with longer tibia.

Bone biomechanical parameters such as breaking force, stiffness, yield load and displacement at yield load are commonly used to determine the bone quality.
Breaking force is the amount of force required to cause a fracture (8). Stiffness is the resistance to elastic deformation and yield load is the flexibility limit point (8, 20). When excessive load is applied than the yield load, permanent deformation occurs in the bones impeding their proper functioning. This means that high breaking force, stiffness and yield load values are important for strong bones in the embryonic period. Metatarsus and tibia of Nick and Brown Nick embryos had the highest breaking force, stiffness and yield load. These results showed that the effect of being brown or white layer hens on examined bone parameters was not statistically significant in the embryonic period. However, genotype is an important parameter for development in the skeletal system in the embryonic period.

For all leg bones, neither genotype nor embryonic age had any significant effect on the displacement values at yield loads. Increased mechanical strength was observed in all genotypes with advancement in embryonic age. Age dependent increase in stiffness and yield load was greatest in metatarsus of Nick embryos, while lowest increase in the stiffness was observed in the femur of Atabey embryos. This means that Nick group had the greatest increment in the mechanical strength of bone with advancement in embryonic age. The embryonic age 
efficiently improved the breaking force, stiffness and yield load in the tibia as compared to the other leg bones.

During embryonic period, minerals for bone development are acquired from the eggshell, albumen and yolk. The strength of the bones depends on their mineral density. In this study, differences were noted in the storage of minerals in different leg bones. The mineral accumulation in the bones of embryos did not differ being they were white or brown laying hens. The effects of the levels of $\mathrm{Zn}, \mathrm{Mn}$ and $\mathrm{Cu}$ on bone development and bone strength have been illustrated in some studies $(4,19)$. Zn deficiency decreases bone collagen turnover and is accompanied by leg deformities $(21,26)$. In the present study it was observed that leg bones of Atak-S embryos had the lowest $\mathrm{Zn}$ content. However, $\mathrm{Mn}$ and $\mathrm{Cu}$ levels of leg bones were not found to be different among the genotype groups. Onbaşılar et al. (16) reported higher $\mathrm{Zn}$ content in the femur of embryos from hybrid type layers as compared to the pure breeds.

Angel (2) reported that tibia is one of the most mineralized bones in the skeleton in the production period. Results in this study showed that tibia and femur were more mineralized bones than metatarsus in the embryonic period. Mineral accumulation in the bones increased from E19 to E21. Only, $\mathrm{Mg}$ level in the metatarsus and tibia were not found statistically different from E19 to E21.

In conclusion, genotype is a factor determining bone development during embryonic period. Bone properties were affected by the genotype but these differences were not related with laying hens being white or brown. The effect of the genotype should be considered in the interaction between embryonic ages. Interaction between genotype and embryonic age should be considered.

\section{Financial Support}

This study was supported by the Ankara University Scientific Research Fund (BAP, 16B0239004).

\section{Ethical Statement}

This study was approved by the Ankara University Animal Experiments Local Ethics Committee (2015/5/102).

\section{Conflicts of Interest}

Authors declare that they have no conflict of interests.

\section{References}

1. Alfonso-Torres KA, Gargaglioni LH, Pizauro JM, et al (2009): Breeder age and bone development in broiler chicken embryos. Braz J Vet. Res Anim Sci, 61, 219-226.

2. Angel R (2007): Metabolic disorders: limitations to growth of and mineral deposition into the broiler skeleton after hatch and potential implications for leg problems. J Appl Poult Res, 16, 138-149.

3. AOAC (2000): Association of Official Analytical Chemists. Official Methods of Analysis of AOAC International. 17th ed, Gaithersburg.

4. Beattie JH, Avenell A (1992): Trace element nutrition and bone metabolism. Nutr Res Rev, 5, 167-188.

5. Boss CB, Fredeen KJ (2004): Concepts, Instrumentation and Techniques in Inductively Coupled Plasma Optical Emission Spectrometry, 3th ed. Perkin Elmer Life and Analytical Sciences, USA.

6. Dawson B, Trapp RG (2001): Basic and Clinical Biostatistics, 3rd ed. Lange Medical Books/McGraw-Hill Medical Publishing Division, New York.

7. Fleming RH, McCormack HA, McTeir L, et al (2006): Relationships between genetic, environmental and nutritional factors influencing osteoporosis in laying hens. Br Poult Sci, 47, 742-755.

8. Forestier-Zhang L, Bishop N (2016): Bone strength in children: understanding basic bone biomechanics. Arch Dis Child, 101, 2-7.

9. Gabrielli MG (2004): Carbonic anhydrases in chick extraembryonic structures: a role for CA in bicarbonate reabsorption through the chorioallantoic membrane. $\mathrm{J}$ Enzym Inhib Med Ch, 19, 283-286.

10. Kierończyk B, Rawski M, Józefiak D, et al (2017): Infectious and non-infectious factors associated with leg disorders in poultry-a review. Ann Anim Sci, 17, 645-669.

11. Korver DR, Saunders-Blades JL, Nadeau KL (2004): Assessing bone mineral density in vivo: Quantitative computed tomography. Poult Sci, 83, 222-229.

12. Kubota M, Abe E, Shinki T, et al (1981): Vitamin D metabolism and its possible role in the developing chick embryo. Biochem J, 194, 103-109.

13. Nasir Z, Peebles ED (2018): Avian embryo nutrition and incubation. Poult Sci, 97, 2994-2995.

14. Onbaşılar EE, Ünal N, Erdem E, et al (2015): Production performance, use of nest box, and external appearance of two strains of laying hens kept in conventional and enriched cages. Poult Sci, 94, 559-564.

15. Onbaşılar EE, Güngör ÖF, Taban S, et al (2018): Comparison of different brown and white layer hybrid embryonic development and uptake of nutrients in the egg. Anim Reprod Sci, 198, 57-64.

16. Onbaşılar EE, Güngör ÖF, Demir T, et al (2018): Femur Properties of Embryo in the Layer Hybrid and Pure Breeds. Braz J Poult Sci, 20, 805-810.

17. Pechak DG, Kujawa MJ, Caplan, AI (1986): Morphology of bone development and bone remodeling in embryonic chick limbs. Bone, 7, 459-472.

18. Sandılands V, Sparks N, Wilson S, et al (2005): Laying hens at depopulation: The impact of the production system on bird welfare. Br Poult Abst, 23- 24.

19. Seo HJ, Cho YE, Kim T, et al (2010): Zinc may increase bone formation through stimulating cell proliferation, alkaline phosphatase activity and collagen synthesis in osteoblastic MC3T3-E1 cells. Nutr Res Pract, 4, 356-361. 
20. Sevil Kilimci F, Kara ME (2013): Basic concepts to assessment of mechanical properties of bones. Animal Health Prod and Hyg, 2, 235-239.

21. Starcher BC, Hill CH, Madaras JG (1980): Effect of zinc deficiency on bone collagenase and collagen turnover. $\mathrm{J}$ Nutr, 110, 2095-2102.

22. Turek SL (1984): Physiology and mineralization of bone. Orthop. 1, 136-190.

23. Whitehead CC, Fleming RH (2000): Osteoporosis in cage layers. Poult Sci, 79, 1033-1041.
24. Whitehead C (2004): Skeletal Disorders in Laying Hens: The Problem of Osteoporosis and Bone Fractures. In: Welfare of the Laying Hen. Poultry Science Symposium Series. CABI Publishing, USA.

25. Yair R, Cahaner A, Uni Z, et al (2017): Maternal and genetic effects on broiler bone properties during incubation period. Poult Sci, 96, 2301-2311.

26. Yair R, Shahar R, Uni Z (2013): Prenatal nutritional manipulation by in ovo enrichment influences bone structure, composition, and mechanical properties. J Anim Sci, 91, 2784-2793. 